\title{
El reconocimiento como ideología 1
}

\author{
AXEL HONNETH* \\ Universidad de Francfurt
}

\begin{abstract}
RESUMEN. El presente artículo se ocupa del problema de si existen formas de reconocimiento moral y social caracterizables como ideológicas, analizando qué propiedades de tales modos de reconocimiento justifican tal calificación. Pretende, además, articular un criterio que permita dirimir normativamente entre formas ideológicas y no ideológicas de reconocimiento. Este criterio será puesto a prueba en el análisis crítico de discursos actuales que reivindican el carácter de reconocimiento social para recientes transformaciones del mercado laboral en el mundo desarrollado.
\end{abstract}

Palabras clave: Filosofía social, Teoría del reconocimiento, Ideología, Filosofía moral.
Abstract. The present article deals with the problem if there are forms of social and moral recognition which can be characterized as ideological. It analyzes which properties of a form of recognition justify such an evaluation and pretends, moreover, to articulate a criterion which permits a normative distinction between ideological and nonideological forms of recognition. This criterion will be put into practice when analyzing contemporary discourses claiming the character of social recognition for recent transformations of the job market in the developed world.

Key words: Social philosophy, Theory of Recognition, Ideology, Moral philosophy

Del mismo modo que en las últimas dos décadas el concepto de reconocimiento se ha convertido en el núcleo normativo de una multitud de esfuerzos políticos emancipatorios, han crecido también las dudas sobre su potencial crítico. A la extensión de este escepticismo teórico ha colaborado sin duda alguna la experiencia de que vivimos hoy en una cultura afirmativa en la que el reconocimiento públicamente manifestado posee con frecuencia un carácter meramente retórico y de sucedáneo: el hecho de ser oficialmente alabado respecto a determinadas cualidades o capacidades parece haberse convertido en un instrumento de la política simbólica, cuya función subterránea es integrar a individuos o grupos sociales en el

1 Versión castellana de José Manuel Moreno Cuevas. Publicado originalmente con el título "Anerkennung als Ideologie" en WestEnd. Neue Zeitschrift für Sozialphilosophie, n 1, octubre 2004, p. 51-70

* Agradezco los valiosos consejos y cuestiones proporcionados por los participantes en el congreso sobre "Power and Recognition" en la Universidad de Utrecht (13-15 de marzo de 2003), en el coloquio "Philosophy and Social Sciences" en Praga (20-24 de mayo de 2004) y en mi propio Coloquio de Investigación sobre Filosofía Social; fueron de especial ayuda las indicaciones realizadas en el marco de tales discusiones por Bert van den Brink, Rahel Jaeggi y David Owen. 
orden social dominante mediante la sugestión de una imagen positiva de sí mismos. Lejos de contribuir eficazmente al mejoramiento de las condiciones de la autonomía de los miembros de nuestra sociedad, el reconocimiento social sirve según todas las apariencias sólo a la generación de actitudes conformes al sistema. Las dudas que por esa razón han sido formuladas entretanto frente al nuevo planteamiento concuerdan con la tesis de que las prácticas de reconocimiento no efectúan un fortalecimiento de los sujetos sino, al contrario, su sometimiento: mediante procesos de reconocimiento mutuo, así se deja resumir la objeción, los individuos son ejercitados en una determinada relación consigo mismos que los motiva para una asunción voluntaria de tareas u obligaciones socialmente útiles (Markell 2003; Oliver 2001; Verweyst 2000).

En esas reservas de principio se refleja la consideración, que ya convenció hace más de treinta años al teórico marxista Louis Alhusser, de que en la praxis del reconocimiento público se localiza el mecanismo estandarizado de todas las formas de ideología (Althusser 1973: 111-172). Su argumento, sólo desarrollado en esbozo y referido exclusivamente a la política de los órganos estatales, fue recuperado después por Judith Butler para consolidarlo en una conceptuación sólida con la inclusión del psicoanálisis de Jacques Lacan (Butler 2001). Althusser se sirve como es sabido del doble significado del concepto francés de "subjectivation" para aclarar su categoría de ideología: los individuos se convierten en sujetos, en el sentido de la adquisición de una conciencia de las propias responsabilidades y derechos, ante todo en la medida en que son sometidos a un sistema de reglas y atribuciones sociales que les otorga identidad social. Pues bien, al ser concebido en esta consideración el acto de reconocimiento según el modelo de la confirmación social, pierde repentinamente lo que podríamos denominar como reconocimiento toda connotación positiva y se convierte en el mecanismo central de toda ideología: reconocer a alguien significa inducirlo, en virtud de requerimientos repetidos y continuados de forma ritualizada, exactamente al tipo de autocomprensión que encaja adecuadamente en el sistema establecido de expectativas de comportamiento.

Ahora bien, no es del todo claro que Althusser haya empleado este concepto de ideología en un sentido crítico; él se limita más bien a un uso puramente descriptivo del concepto (cf. Geuss 1996: cap. I), con el que expone sin valoración normativa la primacía institucional del reconocimiento como mecanismo de producción de sujetos conformes al sistema. Sin embargo, para una teoría crítica de la sociedad que intenta encontrar su fundamento normativo en el cumplimiento del reconocimiento mutuo, las consideraciones de Althusser representan un fuerte reto, pues a la luz de sus reflexiones debe confrontarse con la cuestión de si el reconocimiento social puede asumir efectivamente también la función de aseguramiento del dominio social. En este nuevo contexto el concepto de ideología pierde ciertamente su significado meramente descriptivo y se convierte en una categoría peyorativa; pues ahora son valoradas como ideológicas formas de reconocimiento que por este motivo deben ser consideradas como falsas o injustificadas porque no 
poseen la función de fortalecimiento de la autonomía personal sino la producción de actitudes conformes al dominio (Geuss 1996: 24 ss.).

Naturalmente sería falso suponer que la teoría del reconocimiento no ha tenido costancia desde el principio de fenómenos negativos de sumisión o dominio. En realidad, el impulso crítico de tal teoría se deriva sólo de las manifestaciones sociales de reconocimiento fallido o defectuoso: la mirada debe dirigirse a las prácticas de humillación o envilecimiento a través de las cuales les es escatimada a los sujetos una forma fundada de reconocimiento social y con ello una condición decisiva de la formación de su autonomía (Honneth 2000). Por otra parte, tales expresiones dejan también claro que el reconocimiento, desde un punto de vista conceptual, debería ser tratado como lo contrario de las prácticas de dominio o sumisión. Estas formas de ejercicio de poder deben ser concebidas como fenómenos de reconocimiento escatimado, de menosprecio y humillación calculados, de modo que el reconocimiento mismo nunca pueda caer en la sospecha de ser funcional a un medio de dominación. Esta presunción de inocencia pierde sin embargo toda su evidencia con las reflexiones sugeridas por el concepto de ideología de Althusser, pues con ello aparecen a la luz formas de reconocimiento que son efectivas como medios del dominio social porque producen, según el modelo de la confirmación ritual, una imagen de sí mismo conforme con la sociedad y por consiguiente contribuyen a la reproducción de las relaciones de dominio existentes. Sin dificultad se dejan citar del pasado social ejemplos que hacen transparente cuán frecuentemente el reconocimiento otorgado públicamente sirvió en realidad sólo a la generación y mantenimiento de una forma de autorrelación individual que se ajustaba exactamente al sistema de división dominante del trabajo: el orgullo que el "tío Tom" siente como respuesta a la reiterada exaltación de sus virtudes serviles lo hace un súbdito complaciente en la sociedad esclavista (cf. Raeithel 1995: cap. XXXI); la agitadora apelación a la buena madre y ama de casa pronunciada durante siglos desde las iglesias, los parlamentos o los medios de masas hizo a las mujeres perseverar en una imagen de sí mismas que satisfacía de la mejor manera a la división sexual del trabajo (cf. Hausen 1976: 363-393; Kühn 1993: 43-66); y el aprecio público que disfrutó el soldado valiente y heroico generó continuamente en considerable número una clase de hombres que participó voluntariamente en la guerra a la búsqueda de fama y experiencia (Schilling 2002). Aunque estos ejemplos parezcan triviales, expresan sin embargo con contundencia y claridad que el reconocimiento social puede siempre tener la función de actuar como ideología generadora de conformidad: la repetición continuada de las mismas fórmulas de reconocimiento alcanza sin represión el objetivo de producir un tipo de autoestima que provee de las fuentes motivacionales para formas de sumisión voluntaria.

Sin duda los casos mencionados deben su fuerza sugestiva sólo a la circunstancia de que están investidos de la certeza de una valoración retrospectiva. La elección de los mismos ejemplos así como los medios de su descripción son el resultado de un juicio moral que sólo puede ser admitido desde la perspectiva de 
un presente moralmente más desarrollado: nosotros, que somos contemporáneos de una época que se tiene por moralmente reflexiva frente al pasado, estamos seguros de que la valoración del esclavo virtuoso, de la buena ama de casa y del heroico soldado, tuvo un carácter puramente ideológico; si nos desplazamos por el contrario al pasado correspondiente, parece incomparablemente más difícil diferenciar entre una forma de reconocimiento falsa, ideológica, y una forma correcta, exigida moralmente. Repentinamente se desdibujan los criterios que antes nos parecían seguros: ¿por qué no puede pensarse que el esclavo conseguía, mediante la experiencia de ser apreciado por su dueño blanco por su sumisión, una forma de autoestima que lo ayudaba hasta cierto punto a conseguir autonomía interior? ¿Y no han sido las mujeres compensadas parcialmente de la desestimación de la que fueron objeto por la privación de roles y empleos externos al hogar gracias a que encontraron reconocimiento público como madres solícitas? Finalmente, el esquema valorativo del heroísmo masculino puede haber proporcionado a aquellos hombres que han sufrido la carencia de significación social en sus culturas locales, debido al desempleo o a la falta de cualificación, una subcultura masculina en la que pudieron conseguir prestigio y reputación compensatorios. Estas posibilidades de interpretación dejan claro que un dispositivo determinado de apreciación del valor, que retrospectivamente tomamos por pura ideología, puede ser mostrado en una consideración más cercana de las condiciones históricas como una condición para la adquisición por parte de grupos específicos de una más elevada valoración de sí mismos. Por consiguiente, la determinación del contenido ideológico de las formas de reconocimiento resulta tanto más difícil cuanto más intensamente nos colocamos en las condiciones socioculturales que dominaron en cada momento del pasado. Sólo en el caso en que los afectados mismos se rebelaron contra una práctica dominante de reconocimiento encontramos la base para poder hablar de mera ideología en relación a esa época específica. Pero en general esta dificultad de la valoración pierde su peso con la distancia temporal que nos separa de los casos tratados, pues cuanto mayor es la distancia temporal más claramente dispondremos de criterios aceptados universalmente que nos permitan la distinción entre formas de reconocimiento ideológicas y exigidas moralmente.

Está claro que este problema teórico conserva para el presente su carácter intrincado. Mientras no tengamos ninguna constatación empírica de que los afectados mismos experimentan determinadas prácticas de reconocimiento como represivas, restringidas o estereotipadas, es extremadamente difícil realizar una distinción con sentido entre formas de reconocimiento ideológicas y legítimas. La dificultad surge de que nosotros caracterizamos siempre como acto de reconocimiento la afirmación pública de un valor o de una capacidad que debe corresponder a una persona o un grupo social; hablar de una ideología en un contexto tal significa por tanto atribuir a una praxis determinada en sí positiva la cualidad negativa de ser una sujeción sin represión, a pesar de que parece faltarle prima facie todo carácter discriminador. La cuestión, por consiguiente, se plantea así: 
¿cómo pueden poseer en el presente formas de afirmación pública de un valor social, por lo tanto de reconocimiento, simultáneamente un carácter de dominio? Este es el problema del que quiero ocuparme a continuación. De entrada es recomendable resumir de nuevo lo que entendemos hoy, tras una serie de intentos de aclaración, por una praxis de reconocimiento; con ello se mostrará que el concepto posee un contenido normativo en tanto que indica el comportamiento racional con el que podemos reaccionar a las cualidades valiosas de una persona (o grupo) (I). Esta reflexión conceptual previa ofrece sin embargo sólo una solución aparente al problema de distinguir entre formas de reconocimiento social ideológicas y moralmente justificadas; pues se mostrará que también las ideologías del reconocimiento sólo raramente son simplemente irracionales, sino que en general movilizan fundamentos evaluativos que están situados en el interior de nuestro horizonte valorativo (II). Por ello la solución de nuestro problema sólo puede consistir en el intento de explicitar con detalle las condiciones de aplicación de las formas de reconocimiento hasta que pueda mostrarse el núcleo irracional de todas las ideologías del reconocimiento; presumo que esa irracionalidad no reside en la superficie semántica de los vocablos evaluativos mismos sino en la discrepancia entre promesa evaluativa y realización material (III).

\section{I}

En cierta manera, el problema que quiero colocar en el centro de mi discusión no puede existir para Althusser de ningún modo. Su concepto de reconocimiento es de tal manera unidimensional que no tolera distinciones entre "correcto" y "falso", entre "justificado" e "ideológico"; antes bien para él toda forma de reconocimiento debe poseer el carácter de ideología, porque a través de la sola exhortación o "interpelación" de los destinatarios es impuesta constantemente una unidad imaginaria, que por sí mismos en tanto individuos no deberían poseer. Frente a esto, el intento de diferenciar entre formas de reconocimiento ideológicas y adecuadas debe empezar con la determinación del significado positivo del reconocimiento (cf., para lo siguiente, Honneth 2004a). A pesar de que la bibliografía de investigaciones sobre el tema del reconocimiento se ha ampliado fuertemente en los últimos años, es verdad que el contenido central del referido fenómeno es todavía discutido con intensidad; en la mayoría de los casos el concepto, con una laxa vinculación con Hegel, es vagamente utilizado para actitudes o prácticas a través de las cuales sujetos individuales o grupos sociales son ratificados en algunas de sus cualidades. Sin embargo, queda por aclarar no sólo la relación con el concepto kantiano de "respeto", sino que entretanto se ha tornado también más claro que el concepto de reconocimiento abarca en inglés, francés y alemán elementos de significado diferentes, cuya relación en efecto no es transparente. Así, el concepto parece señalar en alemán sólo aquel contenido normativo vinculado con el otorgamiento de un estatuto positivo, mientras que en inglés y en francés 
abarca adicionalmente todavía el significado epistemológico del "reconocer" o "identificar" a algo o a alguien como tal; a esta dificultad se añade que el concepto puede ser aplicado en los tres idiomas también para los actos de habla de la "confesión" o del "conceder" o "admitir" [Einräumen], donde el "reconocer" recibe primariamente un sentido de pura referencia a sí mismo (Margalit 2001). Finalmente ha entrado en competencia con el modo hegeliano de aplicación del concepto una perspectiva interpretativa derivada de Wittgenstein en la que el reconocimiento hace las veces de reacción performativa a las exteriorizaciones vitales de las otras personas; sobre todo a través de los escritos de Stanley Cavell, los cuales no recurren a Hegel, la categoría de "acknowledgement" ha penetrado por ese camino en el círculo interior de la filosofía analítica (Cavell 2002).

A esta espesura de marañas conceptuales y preguntas sin respuesta sólo puede hoy aportarse claridad mediante una fijación categorial que no se asusta de delimitaciones y restricciones; así hay que tener en cuenta el hecho de que el reconocimiento debe consistir en un acto moral, que está anclado como acontecimiento cotidiano en el mundo social. Parto de cuatro premisas sobre las cuales me parece que existe mientras tanto un amplio consenso: primero, se puede afirmar que el modo original de reconocimiento consiste en lo que el significado alemán de la palabra ubica en el centro: como tal debe ser comprendido ante todo la afirmación de las cualidades positivas de sujetos o grupos, sin que haya que excluir por ello que se pueda establecer una vinculación sistemática con los otros significados. Segundo, parece existir hoy también acuerdo en subrayar el carácter de acción del reconocimiento: un acto de reconocimiento no puede agotarse en meras palabras o declaraciones simbólicas porque es ante todo mediante el correspondiente modo de comportamiento como es generada la credibilidad que para el sujeto reconocido es de importancia normativa. En tanto que nos limitemos a las relaciones intersubjetivas debe hablarse en virtud de ello del reconocimiento como un comportamiento, como una actitud eficaz en el plano de la acción (cf. Ikäheimo 2002; Laitinen 2002). Además, de ello resulta en tercer lugar que tal acto de reconocimiento representa un fenómeno distintivo en el mundo social, el cual por este motivo no cabe ser comprendido como producto derivado de una acción orientada en otra dirección, sino que se debe concebir como expresión de una intención independiente; ya sea en gestos, actos de habla o medidas institucionales, sólo nos las habemos en tales manifestaciones o medidas con un caso de reconocimiento cuando su propósito primario de alguna manera está dirigido positivamente a la existencia de otra persona o grupo. Esta decisión teórica previa excluye por ejemplo concebir como formas de reconocimiento actitudes positivas que acompañan forzosamente el seguimiento de toda una serie de intereses diversos de interacción: si tengo el fuerte deseo de jugar regularmente al ajedrez con una determinada persona, alcanza así expresión probablemente una apreciación especial de sus capacidades intelectuales, pero el fin primario de la intención de mi acción está orientado a jugar juntos al ajedrez. Una cuarta premisa sobre la que hoy existe acuerdo se deja resumir en la tesis de que el reconocimiento representa un 
concepto genérico que abarca diferentes subtipos: así por ejemplo en las conductas del amor, del respeto jurídico y de la apreciación del valor resaltan en cada caso diferentes acentuaciones de una actitud fundamental que se deja conceptuar genéricamente como reconocimiento.

Las cuatro premisas que he expuesto hasta ahora resumen solamente de dónde tiene que partir hoy un uso conceptual medianamente claro: el reconocimiento debe ser concebido como género de diferentes formas de actitud práctica en la que cada vez se refleja el objetivo primario de una determinada afirmación del que está enfrente. A diferencia de lo que Althusser sostuvo, tales comportamientos afirmadores poseen un carácter indudablemente positivo, porque permiten al destinatario identificarse con sus cualidades y con ello alcanzar una mayor autonomía; lejos de representar una mera ideología, el reconocimiento configura las condiciones previas intersubjetivas de la capacidad de realizar autónomamente los propios objetivos vitales ${ }^{2}$. Sin embargo, el auténtico desafío para la clarificación conceptual comienza primeramente allí donde se trata del carácter epistemológico de tal comportamiento afirmativo; este punto está demarcado por la cuestión de si debemos comprender el reconocimiento más bien como un acto atributivo o como uno receptivo. En la cuestión de cómo debemos caracterizar adecuadamente el caso genérico del reconocimiento parece que nos encontramos ante dos alternativas que conciernen a la relación cognitiva con el compañero de interacción: o bien podemos concebir la afirmación que acontece mediante un acto de ese tipo según el modelo de una atribución, en virtud de la cual puede imputársele en cierto modo al otro sujeto una cualidad nueva y positiva; o bien comprendemos ese acto desde el modelo de la percepción, de manera que la cualidad ya existente de una persona es de algún modo confirmada o públicamente afirmada sólo de forma secundaria. En el primer caso, lo que entendemos como reconocimiento añadiría o prestaría al sujeto en cuestión un estatuto que no poseía antes; mientras que en el segundo caso se trataría de una determinada clase de percepción de un estatuto ya existente de manera independiente. Otra posibilidad de determinar las diferencias entre esas dos maneras de ver podría consistir en hablar, en el primer caso, de una realización productiva del reconocimiento y, en el segundo, de una realización solamente reproductiva: el estatuto o las cualidades positivas que posee una persona o un grupo social son o bien generadas o bien restituidas mediante el acto de reconocimiento de una manera determinada y en todo caso significativa.

${ }^{2}$ Aquí se muestra claramente una diferencia con Althusser que descansa tan profundo en lo ontológico-social que difícilmente permite aquí una discusión adecuada: por razones de principio Althusser no concede a los sujetos la oportunidad de ganar un mayor grado de autonomía en la ejecución de sus acciones y decisiones vitales; él parte más bien de que los individuos sólo pueden convertirse en sujetos capaces de identificación social en la medida en que mediante su apelación pública son sometidos a una red de normas sociales que no concede ningún margen a la variación respecto a la autonomía individual. Frente a esto parto (con Durkheim) de que precisamente las reglas sociales del reconocimiento deben permitir distinguir qué espacios abren a la autonomía individual. 
No es fácil decidirse entre estos dos modelos de representación alternativos porque cada uno de ellos parece poseer una virtud diferente. Si mi visión es adecuada, el modelo de la percepción o recepción hace justicia a nuestra intuición de que el comportamiento de reconocimiento debe tratarse de un acto que está motivado por razones prácticas, a saber: en el reconocimiento reaccionamos de manera correcta o adecuada a aquellas razones que son erigidas por las cualidades evaluativas que los seres humanos poseen previamente de diversos modos (Laitinen 2002). En cambio, el modelo que opera con la representación de una "atribución" está libre de mezcla con tal realismo de los valores; aquí se rinde cuentas a la intuición de que el reconocimiento debe tratarse de un acto constituyente cuando se atribuyen capacidades particulares a una persona o un grupo. El inconveniente de esta concepción se encuentra efectivamente en el punto en que parece encontrarse la virtud del modelo de la recepción: si a través del comportamiento reconocedor de otro sujeto deben ser meramente atribuidas cualidades positivas, entonces no poseemos ningún criterio interno para juzgar acerca de la corrección o adecuación de una tal atribución; más bien al marco de variación del reconocimiento no se le puede establecer ningún límite porque debemos considerar toda cualidad como posible capacidad o elemento definidor del estatuto de una persona en tanto que sólo llega a ser efectivo a través de un acto de atribución. En este punto, sólo ofrecería una salida la tesis de que la legitimidad del reconocimiento se limita a la cualidad normativa de su puesta en práctica, pero entonces perdería el concepto de reconocimiento todas sus implicaciones morales que precisamente deben hacerla diferenciable de una mera propuesta de etiqueta sociológica.

Ahora bien, a primera vista tampoco la perspectiva opuesta, a saber, el modelo de la recepción o de la respuesta, queda mucho mejor situada. Para poder sostener que en el comportamiento de reconocimiento se reacciona correctamente a las cualidades valiosas de una persona o de un grupo, debe ser presupuesta la existencia objetiva de valores de una manera que ya no es compatible con nuestra comprensión de su carácter constituido. A decir verdad, parece correcto que tendríamos que afincar el reconocimiento en el ámbito de las razones, para que no se le dé bajo cuerda el carácter de una acción moral; pues sólo cuando nuestro reconocimiento de otra persona está motivado mediante razones, las cuales, dado el caso, también podríamos intentar articular, se deja concebir como una acción de discernimiento y con ello incluir en un sentido más amplio en el ámbito de la moral. También la propuesta adicional de caracterizar ese tipo de razones como "evaluativas" debe parecer evidente en cuanto que en el comportamiento de reconocimiento parecemos siempre atestiguar el valor de una persona (o grupo); las limitaciones morales a las cuales nos sabemos obligados en el reconocimiento, resultan de las cualidades valiosas que en cierto modo llevamos a expresión pública mediante nuestro comportamiento recognoscente. La dificultad comienza precisamente en el momento en que se trata de determinar con más precisión el estatuto de tales razones evaluativas. Aquí parece que no se ofrece otra salida que recurrir a un realismo del valor que no es ya conciliable con el resto de nuestras 
convicciones ontológicas de fondo. Esta situación embarazosa cambia desde luego si admitimos la posibilidad de que semejantes valores exponen certezas del mundo de la vida, cuyo carácter puede estar sujeto a alteraciones históricas; no serían cualidades de valor fijas, objetivas, sino variables históricamente, que debemos poder percibir en personas (o grupos) para reaccionar a ellas correctamente en un comportamiento de reconocimiento. La imagen esbozada de este modo debería ciertamente ser completada todavía con elementos adicionales para conseguir una teoría más o menos plausible: el mundo social de la vida podría ser concebido al modo de una "segunda naturaleza", en la que los sujetos son socializados de tal modo que sucesivamente aprenden a experimentar las cualidades valiosas de las personas; este proceso de aprendizaje debería ser concebido como un curso complejo, en tanto que en él con la percepción de cualidades evaluativas serían adquiridos al mismo tiempo también los modos de comportamiento correspondientes, cuya peculiaridad debería consistir evidentemente en la restricción de nuestro egoísmo natural; en consecuencia, podríamos comprender el comportamiento de reconocimiento humano como un haz de hábitos que en el proceso de socialización han sido vinculados con las razones capaces de constatar el valor de otras personas (McDowell 2002; Lovibond 2002, Parte II; Honneth 2003).

En todo caso, con estas consideraciones no está resuelto todavía el problema que parece constituir la auténtica dificultad de un tal realismo moderado de los valores. De las cualidades valiosas que podemos reconocer adecuadamente en otras personas se había dicho que sólo pueden poseer realidad efectiva en el horizonte de experiencia de un mundo de vida determinado; cuando sus miembros han sido socializados exitosamente en su cultura, perciben tales cualidades de valor personales del mismo modo que realidades objetivas del mundo social circundante, tal como experimentan también otras particularidades culturales primeramente como hechos dados evidentes. Con ello surge también en el marco de esta concepción el peligro de un relativismo que no es compatible con los fines normativos del concepto de reconocimiento; pues los valores a los que se ajustaría la adecuación del comportamiento de reconocimiento parecen poseer validez normativa en cada caso sólo para una única cultura. En consecuencia, el relativismo que acompaña al modelo de la respuesta o de la recepción no se podría distinguir de ningún modo del correspondiente al modelo de la atribución: en ambos casos la validez de los comportamientos de reconocimiento, tanto si se los describe como atribuciones o como reacciones adecuadas, sería dependiente solamente de las realidades normativas de cada forma de vida. Para el modelo de la recepción esta dificultad puede ser sólo esquivada, según mi convicción, cuando el realismo moderado del valor es pertrechado con una robusta concepción del progreso; en el fondo esto significaría suponer un desarrollo orientado que permitiría juicios fundamentados sobre la validez transhistórica de cada cultura de reconocimiento (cf. sobre esto Honneth 2003). Sin poder entrar aquí en los detalles de una concepción tal del progreso, que según mi convicción debería ser 
determinada como forma de reflexión sobre nuestro saber de orientación en el mundo de la vida (cf. Honneth 2004b), quiero limitarme a la indicación del pensamiento central: con la diferenciación histórica de cualidades de valor, que gracias a nuestra socialización como sujetos humanos aprendemos a percibir y a considerar racionalmente, se eleva al mismo tiempo el nivel normativo de nuestras relaciones de reconocimiento; pues con cada valor que confirmamos mediante reconocimiento crece la posibilidad para los seres humanos de identificarse con sus capacidades y de alcanzar correspondientemente una mayor autonomía. Esta indicación debe bastar para justificar la tesis del anclaje del concepto de reconocimiento en un realismo moderado del valor.

Pero antes de poder volver a la cuestión de cómo podemos diferenciar formas ideológicas y justificadas de reconocimiento, debe ser tratado todavía brevemente al menos un problema adicional. Surge de la circunstancia de que la mayoría de las veces se habla de ideologías como formaciones de conciencia o sistemas de enunciados evaluativos cuya fuente no es la relación intersubjetiva, sino reglas o acuerdos institucionales; con Marx, que tomó la forma del contrato burgués por una tal institución productora de ideología (Marx 1971: 181-191), partimos de que la condición específica de determinadas instituciones es lo que conduce causalmente al surgimiento de convicciones engañosas o ficcionales. Si, por lo tanto, también deben poderse formar modelos de reconocimiento ideológicos en tal sentido, debemos en consecuencia aclarar qué significa que pueden donar reconocimiento no sólo personas sino también las instituciones sociales; desde plano del reconocimiento intersubjetivo, al cual nos hemos orientado hasta ahora, debemos transitar hacia el plano del reconocimiento garantizado institucionalmente (cf. Renault 2004).

El punto de partida de una transición tal la constituye la observación de que también las regulaciones y prácticas institucionales pueden contener representaciones acerca de qué cualidades de valor de los seres humanos deben alcanzar reconocimiento de un modo específico. Así, por ejemplo, en la instituición de la pequeña familia moderna alcanza expresión el valor que debe poseer de modo reconocido el ser humano en tanto sujeto de necesidades privado, mientras que en el principio institucionalizado de igualdad del derecho moderno ha encontrado su plasmación el hecho normativo de que los integrantes de las sociedades modernas deben ser respetados como sujetos iguales y libres; en ambos casos se puede concebir la institución correspondiente casi como una encarnación institucional de formas específicas de reconocimiento que presentan a los sujetos intersubjetivamente a partir de sus específicas cualidades de valor. Pero de tales instituciones, en las que alcanzan expresión modelos de reconocimiento social, deben distinguirse las prácticas y regulaciones institucionales que sólo indirectamente o como efecto secundario articulan determinadas formas de reconocimiento; en la práctica rutinaria de casi todas las instituciones se hace uso de determinadas representaciones acerca de los sujetos humanos que, aunque deben donar un reconocimiento no intencional, pueden ser concebidas sin embargo como cristalizaciones de modelos 
de reconocimiento. Así se reflejan formas reñidas de reconocimiento social tanto en las regulaciones que establecen la remuneración, la protección frente a enfermedades o el tiempo de vacaciones de los trabajadores en determinados ramos como por ejemplo en las prácticas y rutinas organizatorias con las que los pacientes son tratados en los hospitales; los esquemas de percepción y comportamiento que forman las condiciones previas para que los individuos sean tratados en tales organizaciones de una determinada manera como miembros o clientes pueden ser concebidos como sedimentaciones de prácticas de reconocimiento del mundo de la vida. Verdad es que la dirección de tales sedimentaciones puede también invertirse, como cuando las organizaciones asumen un papel prioritario en la producción o descubrimiento de nuevas cualidades de valor de los seres humanos; en tales casos se imponen modelos de reconocimiento modificados primeramente en las regulaciones y prácticas de una institución antes de que alcancen expresión en la praxis narrativa de un mundo de la vida. Probablemente es este segundo caso de reconocimiento institucional de especial importancia para la cuestión de en qué medida determinados modelos de reconocimiento poseen un carácter ideológico porque despiertan una disposición para la sumisión voluntaria.

\section{II}

En las reflexiones que he realizado hasta ahora he afrontado esencialmente la cuestión de la adecuada comprensión del concepto de reconocimiento. Ante la alternativa planteada entre un modelo de la atribución y un modelo de la recepción, he tomado el camino de un realismo moderado del valor: por reconocimiento debemos entender un comportamiento de reacción con el que respondemos de manera racional a cualidades de valor que hemos aprendido a percibir en los sujetos humanos conforme a la integración en la segunda naturaleza de nuestro mundo de la vida. Esta determinación del concepto ostenta suficientemente la distancia que lo separa de la propuesta de Althusser de que toda forma de reconocimiento representa sin distinción una forma de ideología, que arraiga en la idea de que independientemente de en tanto qué sean apelados en cada caso los sujetos, la mera imputación de un estatuto social representaría ya una praxis ideológica, porque ella, con la ilusión de la unidad y la identidad, produciría al mismo tiempo la disposición a asumir las correspondientes expectativas de comportamiento. En cambio, la propuesta desarrollada aquí se basa en la posibilidad de una forma racional, adecuada, de reconocimiento que consiste en hacer valer públicamente de modo performativo cualidades de valor ya existentes de los seres humanos. En todo caso, esta formulación no da a conocer todavía claramente de manera suficiente por qué un concepto de reconocimiento como éste tiene que significar un comportamiento moral; aunque tenemos que ver aquí con una acción mediada por razones evaluativas, ella sola sin embargo no muestra todavía que deba tratarse de un acto moral. La conexión referida aparece cuando se considera de 
manera más precisa ese aspecto que antes describí como restricción del egocentrismo. En cierto modo, este pensamiento se enlaza con Kant, que en el contexto de la introducción de su concepto de respeto sostuvo que "cada representación de un valor" nos impele a imponer una limitación a nuestra acción que efectúa "una suspensión de nuestro amor a nosotros mismos" (Kant 1999: 20 ss., nota 2, A2: 401): se puede sostener entonces que reconocer a alguien significa percibir en él una cualidad de valor que nos motiva intrínsecamente a comportarnos ya no de manera egocéntrica sino adecuada a los propósitos, deseos o necesidades de los demás. Con esto se torna claro que el comportamiento de reconocimiento debe constituir por este motivo una acción moral porque se deja determinar por el valor de las otras personas; el comportamiento de reconocimiento se orienta no según los propios propósitos sino según las cualidades evaluativas de los demás. Si esto es así, deben poderse distinguir del mismo modo tantas formas de acción moral como valores a reconocer en los sujetos humanos. Por eso he llegado a la conclusión en algunos de mis recientes trabajos de distinguir tres fuentes de la moral, que tienen que corresponder a las formas diferenciadas de reconocimiento de nuestro mundo de la vida; pues, como yo sostendría en concordancia con una serie de otros autores, el horizonte de valores de las sociedades modernas está marcado por la representación de que los seres humanos deben recibir valor al menos como seres necesitados, con iguales derechos en su autonomía y eficientes, al cual corresponden las distintas formas de comportamiento de reconocimiento (amor, respeto jurídico, valoración social) (Honneth 2003a).

Antes de continuar con esta línea de pensamiento, quisiera primero tornar a la cuestión que propiamente está en el centro de mi escrito. No hay que excluir, así dije al comienzo, que las formas de reconocimiento social posean sólo una función ideológica, porque pueden incitar en el individuo una relación consigo mismo que hace juego con el orden de dominio existente; en efecto, en lugar de dar expresión a un valor, tales ideologías del reconocimiento proporcionarían la disposición emocional para cumplir sin resistencia las tareas y obligaciones esperadas. Posiblemente tenga sentido aquí primeramente seguir restringiendo el círculo de declaraciones valorativas públicas y representaciones del sujeto que es relevante para tal papel ideológico en general; pues la mayoría de las clasificaciones evaluativas que podemos encontrar en nuestro mundo de la vida no cumplen en absoluto las precondiciones para poder ser consideradas como ideologías del reconocimiento.

Los sistemas de persuasión que pueden ser considerados como tales ideologías deben poseer en primer lugar evidentemente la cualidad de dar expresión al valor de un sujeto o de un grupo de sujetos, es decir, la función que se espera de ellos sólo pueden cumplirla si dan a los individuos la oportunidad de referirse a sí mismos de manera positiva de tal modo que se vean alentados a la asunción voluntaria de determinadas tareas. En tal caso, se excluyen del círculo de declaraciones valorativas aquí determinadas todas las clasificaciones que poseen un carácter discriminatorio explícito; aquellos sistemas de persuasión en los que, 
como el racismo, la misoginia o la xenofobia, se deniega un valor a grupos de personas específicos, no pueden asumir en absoluto el papel de ideologías del reconocimiento porque normalmente conducen a dañar la imagen de sí mismos de las personas apeladas. Las ideologías que deben ser efectivas en virtud del reconocimiento social no pueden contribuir a la exclusión de los grupos de personas correspondientes sino a su integración.

En segundo lugar, para conseguir ese efecto los sistemas de persuasión indagados deben ser para los propios afectados dignos de crédito; ellos yerran su función performativa cuando las personas apeladas no encuentran ninguna buena razón para identificarse con la declaración valorativa vigente para ellos. Esta condición restrictiva tiene no sólo una cara trivial sino también una compleja, que no es fácil de aclarar. Naturalmente todas las declaraciones valorativas positivas capaces de reforzar la imagen de sí mismo de una persona o de un grupo de personas deben ser realistas en el sentido habitual de la palabra de manera que se refieran de forma significativa a capacidades o virtudes efectivamente dadas: elogiar a un policía por su habilidad para el cálculo puede ser tan poco razonable como alabar a un matemático talentoso por su fuerza física, pues ambos serían distinguidos por méritos extraños a sus respectivos oficios. Pero más esencial que este componente realista hay en el criterio de la credibilidad aún un segundo componente que está unido a la progresión del ámbito de las razones evaluativas: sólo se vuelven aptas para ser aceptadas por las personas apeladas aquellas declaraciones valorativas que no permanecen rezagadas en niveles evaluativos que ya se han abandonado a través del proceso de superación de las atribuciones de identidad unidimensionales o inadecuadas. Expresado en términos positivos y más sencillos, de esto resulta que las ideologías del reconocimiento sólo pueden utilizar las declaraciones valorativas que de alguna manera están a la altura del vocabulario evaluativo de cada presente; por el contrario, las declaraciones que señalan como visibles cualidades de valor que entretanto han caído en descrédito, serán percibidas por los sujetos como carentes de credibilidad. En todo caso, es inherente al criterio de la credibilidad, junto al componente de realidad, un componente de racionalidad, que posee de manera inequívoca un índice histórico o temporal: una mujer que aún hoy es alabada por sus virtudes como ama de casa posee pocas posibilidades de identificarse con tal declaración valorativa hasta el punto que vea aumentar duraderamente su autoestima.

En concordancia con esto, no podemos caracterizar en realidad tales modelos de reconocimiento, que podemos describir como "normalizadores" (Emcke 2000; Rösner 2002), como ideologías del reconocimiento; pues con la expresión "normalización" se quiere decir en este contexto que una persona o un grupo es reconocido mediante la aplicación de determinaciones de cualidades o atribuciones de identidad que son experimentadas por las personas o los miembros del grupo como restricción del espacio de juego de su autonomía (Emcke 2000: 237 ss.). Esto significa que un reconocimiento normalizante no puede motivar el desarrollo de una imagen de sí mismo positiva que conduzca a una asunción voluntaria 
de tareas y privaciones decididas por otros. Sin duda alguna, el caso es más difícil cuando sólo suponemos el efecto de una normalización sin poseer en cambio el punto de apoyo del malestar o la protesta de los afectados mismos; en tales situaciones la caracterización negativa está vinculada con la hipótesis de que los afectados deberían, a partir de un conocimiento completo de todas las circunstancias, rechazar las determinaciones de cualidades correspondientes porque con ellas son coartados en su autonomía. En el fondo, esa suposición va a parar a la tesis de que un determinado modelo de reconocimiento actúa como "normalizante" cuando una atribución restrictiva y evaluativamente anticuada de identidad es conservada de manera injustificada, mientras que las ideologías del reconocimiento sólo podrían mantener su efecto libre de represiones con la ayuda de declaraciones valorativas contemporáneas y racionales desde una perspectiva evaluativa.

Quizá puede nombrarse aún una tercera condición que debe darse para que formas de reconocimiento social puedan asumir una función ideológica: tales declaraciones valorativas no deben ser sólo positivas y dignas de crédito, sino que deben ser también contrastantes, de manera que en cada caso den expresión a nuevos valores o capacidades específicas: esta restricción se deriva de que los individuos sólo tienen la posibilidad de identificarse con las determinaciones válidas para ellos cuando a su luz pueden experimentarse como distinguidos; por eso las declaraciones valorativas, que deberían poder aplicar sobre sí, deberían mostrar un contraste en comparación con el pasado o con el orden social circundante, lo cual ofrece una garantía para la experimentación de distinciones positivas específicas. Si en cambio una forma existente de reconocimiento social es extendida simplemente a un medio social que hasta el momento había estado excluido de ella, debería faltar en ese momento el contraste decisivo que despierta la disposición motivacional para la sumisión voluntaria.

Con estas limitaciones, que poseen claramente distinto peso, he esbozado por el momento solamente las condiciones bajo las cuales pueden ser exitosas las ideologías del reconocimiento: están en condiciones de cumplir con la función de promover una relación individual consigo mismo que motive a la asunción voluntaria de tareas y funciones cuando las declaraciones valorativas vinculadas con ellas sean al mismo tiempo positivas, dignas de crédito y en alguna medida contrastantes. Pero tomadas en conjunto, esas condiciones de éxito dejan al mismo tiempo también claro que tales ideologías del reconocimiento no pueden ser concebidas simplemente como sistemas de persuasión irracionales; más bien deben movilizar razones evaluativas que posean bajo las condiciones dadas la suficiente fuerza de convicción para poder motivar racionalmente a las personas apeladas a aplicar sobre sí tales razones. Al contrario de las ideologías excluyentes, que por así decirlo violentan el horizonte de percepción evaluativo del presente, en tanto que se tornan ciegas para determinadas cualidades de valor de grupos de personas, las ideologías del reconocimiento operan en el marco de las razones históricamente existente: amplían de alguna manera sólo las cualidades evaluativas que 
hemos aprendido a percibir en seres humanos hacia un nuevo significado que en efecto posee, en su aplicación exitosa, la cualidad de producir una relación consigo mismo ajustada a la función y adaptativa. Como todo cambio de acento del reconocimiento social, tales ideologías racionales están también sujetas al horizonte de valor que abarca la cultura normativa del reconocimiento en las sociedades modernas; de ahí que tampoco ellas puedan menos que servirse semánticamente de esos principios del amor, de la igualdad jurídica o de la justicia respecto al rendimiento que organizan hasta en nuestra percepción evaluativa las relaciones existentes del reconocimiento recíproco. Por consiguiente, debe preguntarse, ¿cómo pueden diferenciarse bajo estas condiciones formas justificadas e injustificadas de reconocimiento social? Para decirlo de manera penetrante, ¿cuándo se convierte un cambio de acento del reconocimiento en una ideología que sólo posee el efecto de promover una relación consigo mismo adecuada a una función?

El alcance de las dificultades que se presentan aquí se hace patente desde luego en primer lugar cuando nos damos cuenta de que el desarrollo histórico del reconocimiento se efectúa en la forma de apertura de nuevas perspectivas en el horizonte de los principios generales: con la apelación a un principio de reconocimiento ampliado es puesta en juego una nueva singularidad hasta entonces descuidada, cuya consideración impulsa a ampliar el horizonte de percepción evaluativo y con ello a conseguir una intensificación del reconocimiento. Así se puede sostener, según mi opinión, que en los dos últimos siglos con la apelación al significado normativo del "amor" se hicieron valer una y otra vez nuevas necesidades -el bienestar de los niños, la necesidad de autonomía de la mujer, por nombrar solo dos ejemplos-, que han conducido paulatinamente a una profundización de la asistencia y la ayuda mutuas; la misma dinámica puede observarse también naturalmente en las relaciones de reconocimiento del derecho moderno, en las cuales el proceso de reclamación judicial respecto a situaciones de la vida hasta entonces descuidadas ha conducido a una elevación indudable de la igualdad jurídica; e incluso en relación al principio del rendimiento yo hablaría de una tal dialéctica de lo general y lo singular, porque aquí una ininterrumpida lucha simbólica por el significado de lo que "rendimiento" o "mérito" deben significar nos ha llevado al umbral de un tiempo en el que comienza a perfilarse una comprensión ampliada de las contribuciones y rendimientos sociales. Cuanto más exactamente constatamos que las relaciones de reconocimiento se han transformado históricamente y se han ampliado y mejorado a propósito de cambios de acento de los principios generales, tanto más difícil se torna identificar en la corriente del flujo de significado formas de reconocimiento meramente ideológicas; pues quién nos dice que una nueva valoración aparentemente ideológica y adecuada a una función no consiste nuevamente sólo en uno de esos desplazamientos de acento a propósito de los cuales progresa históricamente la lucha por el reconocimiento. El caso es sencillo solamente allí donde los afectados oponen resistencia contra una nueva forma de distinción evaluativa; aquí tenemos al menos las primeras razones para cuestionar la legitimidad de las formas 
modificadas de reconocimiento y proclamar la sospecha de ideología. Pero donde tal protesta no aparece, donde los sujetos parecen alcanzar un respeto hacia sí mismos reforzado bajo los efectos de una nueva forma de reconocimiento, nos falta por el momento todo criterio para diferenciar entre desplazamientos de acento ideológicos y justificados. Quiero presentar en la última parte de mi reflexión un ejemplo del pasado reciente para esbozar al menos el contorno de una respuesta a la cuestión.

\section{III}

Se ha mostrado hasta ahora que las ideologías del reconocimiento deben representar clasificaciones positivas, cuyo contenido evaluativo es tan digno de crédito que pueden ser aceptadas con buenas razones por las personas apeladas: su relación consigo mismas debe poder cambiar a la luz de la nueva distinción de tal forma que les espera el premio psicológico de un autorrespeto realzado, cuando de hecho hacen propias las aptitudes, necesidades o virtudes asociadas con la distinción. Los candidatos elementales para una ideología tal parecen serlo los contenidos de publicidad que desarrollan iconográficamente el esquema del reconocimiento de tal modo que un grupo determinado de personas se ve positivamente exhortada a adaptar los modos de comportamiento propios a los estándares ofrecidos; el objetivo deseado es alcanzado cuando la ejecución de la praxis correspondiente sólo es posible con la ayuda de artículos de consumo para los cuales el spot publicitario habría, de manera más o menos velada, hecho publicidad. Ciertamente, el ejemplo de la publicidad es apropiado sólo con reservas para hacer resaltar las cualidades que caracterizan a las ideologías del reconocimiento; pues en general tales contenidos publicitarios son recibidos sólo bajo la reserva mental de ofrecer meras ficciones, de manera que no son capaces de intervenir de modo efectivamente transformador en la praxis vital. Pero al traspasar ese umbral actúan de hecho como formadores de conductas; así ejerce la publicidad de determinados productos el mismo poder que es propio también de las formas ideológicas de reconocimiento: poseen la capacidad "regulativa" de generar modos de conducta que enlazan su realización con el beneficio de la adquisición de autoestima y aprobación pública. El poder que las ideologías del reconocimiento ejercen es por ello al mismo tiempo libre de represión y productivo enteramente en el sentido de Foucault: la disposición favorable para una serie de prácticas y modos de conducta que armoniza con la función de la reproducción de la dominación social surge de la promesa de reconocimiento social para la manifestación subjetiva de determinadas capacidades, necesidades o deseos (Detel 1998: 55 ss.). Pero naturalmente tampoco con esta clarificación conceptual del tipo de poder que representan las ideologías del reconocimiento está todavía respondida la cuestión de cómo pueden ser identificadas como tal en la corriente ininterrumpida de una compleja lucha por el reconocimiento. Aunque la comparación con la 
publicidad moderna hace transparente que tales ideologías deben poseer un carácter apelativo que apoye retóricamente su credibilidad evaluativa, sin embargo tampoco las exigencias razonadas de cambios de acento del reconocimiento social podrán realizarse ya hoy sin elementos de una política simbólica que les proporcione atención pública. En lugar de continuar discutiendo la cuestión de forma sólo conceptual, quiero recurrir a continuación a un ejemplo empírico, de cuya descripción espero una clarificación más precisa. De la multiplicidad de nuevos modelos de reconocimiento que se perfilan hoy en nuestra cultura social -la revaloración del trabajo doméstico femenino en conexión con el principio del rendimiento, la consideración de la pertenencia a minorías culturales en conexión con la igualdad jurídica, la idea de un reconocimiento del "trabajo cívico"-, quisiera escoger un caso que ya de principio posee todos los indicios de una pura ideología del reconocimiento. La discusión del caso debe mostrar si se pueden encontrar criterios que den una segura referencia sobre el contenido ideológico de las formas de reconocimiento.

En los tiempos recientes está teniendo lugar en la esfera laboral del capitalismo altamente desarrollado un profundo cambio estructural a consecuencia del cual los empleados son apelados de un nuevo modo: en la literatura empresarial de nuestros días no se trata ya simplemente de "asalariados" o de "fuerza laboral", sino de "empresarios de la fuerza de trabajo" creativos (Opitz 2004: cap. 8; Voß/Pongratz 1989). El desplazamiento de acento que va acompañado de tal calificación modificada parte del discurso de la autorrealización individual para transferirlo a la organización del trabajo como factor de producción y de prestación de servicios: las crecientes necesidades de autorrealización deberían ser satisfechas en la esfera laboral de manera que a través de la nivelación de jerarquías, el aumento de la autonomía del equipo de trabajo y un grado mayor de capacidad de control mejoraría la posibilidad de comprender la actividad como exteriorización autónoma de capacidades aprendidas. Pero parece además que la nueva apelación va acompañada de una imagen de la profesión completamente modificada, porque los sujetos no deben concebir ya su actividad como cumplimiento de una necesidad sino como realización de una "vocación"; la idea de que los empleados se califiquen a sí mismos de empresarios exhorta a caracterizar cada cambio de lugar de trabajo o cada modificación en las condiciones laborales como resultado de una decisión propia, que se orienta sólo según el valor intrínseco de cada trabajo. De este modo, con la apelación modificada parece imponerse también un cambio de acento en el viejo principio del rendimiento, porque ahora se exige a los empleados dependientes aquellas capacidades autónomas, creativas y flexibles, que hasta el momento habían sido las recomendaciones clásicas reservadas sólo para los empresarios: a cada fuerza de trabajo cualificada, así dice esta nueva forma de reconocimiento, le es posible planear el propio camino profesional como la empresa arriesgada de una utilización autónoma de sus diversas capacidades. 
Naturalmente es factible reconocer en esta apelación modificada de los empleados el esbozo exacto de esa forma de reconocimiento que antes caractericé con pretensión regulativa como ideología. La sospecha que se impone a la luz del ejemplo es que el desplazamiento de acento del reconocimiento cumple aquí primariamente la función de promover una nueva relación consigo mismo que motive a la aceptación voluntaria de cargas de trabajo considerablemente modificadas: la flexibilización y la desregulación del trabajo que acompañan al cambio estructural neoliberal del capitalismo exigen competencias de automercantilización productiva que precisamente pueden ser generadas productivamente mediante la calificación reconocedora como "empresario de la fuerza de trabajo" (Opitz 2004: cap. 8). Pero entre esa sospecha de principio y una afirmación fundamentada existe una brecha teórica cuya superación exige criterios que son difíciles de desarrollar. Si en las nuevas apelaciones debe tratarse de hecho de un reconocimiento, entonces deben ser cumplidas primeramente las condiciones que antes caractericé como cualidades de una ideología tal: los sujetos poseerán buenas razones de tipo evaluativo para aplicar a sí mismos las caracterizaciones modificadas, de manera que eventualmente también alcancen un grado más alto de autoestima o respeto por sí mismos. Por eso queda cerrado el camino para aquella crítica de la ideología que consiste en demostrar la irracionalidad de los sistemas de persuasión tenidos por ideológicos; al menos en sentido evaluativo la nueva forma de reconocimiento debe ser racional hasta tal punto que ella sea percibida por los afectados como suficientemente digna de crédito como para poder aplicarla sobre sí mismos. Por ello me parece aconsejable seguir determinando con precisión las condiciones de cumplimiento del reconocimiento social para en lo posible dar con los déficits que caracterizan a las ideologías del reconocimiento. Para ese fin debo retomar el hilo conductor de mi argumentación donde lo dejé cuando caractericé al reconocimiento como la reacción moral y adecuada desde un punto de vista racional a las cualidades de valor de los seres humanos.

Ya al comienzo de mis consideraciones había dicho que el reconocimiento no debe agotarse en meras palabras o manifestaciones simbólicas, sino que debe acompañarse de acciones acreditadoras: un acto de reconocimiento queda de alguna manera incompleto mientras no desemboque en modos de conducta que pongan de manifiesto también de forma efectiva el valor articulado. En todo caso sólo tiene sentido hablar de cumplimiento del reconocimiento en determinados modos de conducta mientras estamos ante interacciones sencillas en las que dos personas se hallan frente a frente; sin embargo, tan pronto como cambiamos de nivel y tenemos ante nosotros casos de reconocimiento generalizado, como es practicado por instituciones sociales, no debemos suponer el cumplimiento del reconocimiento simplemente en forma de modales o maneras de conducta. Aunque el reconocimiento institucional generalizado se traduce a largo plazo en formas modificadas de hábito, la fuente primaria de su cumplimiento reside sin embargo en el ámbito de las medidas o disposiciones institucionales: cuando son alcanzadas nuevas formas de reconocimiento social 
generalizado deben transformarse disposiciones jurídicas, deben ser establecidas otras formas de representación política y deben emprenderse distribuciones materiales.

Por ello en la credibilidad del reconocimiento social debe ser considerado junto al componente evaluativo también un segundo componente "material", que según el grado de complejidad de la interacción social consiste o bien en modos de conducta apropiados o en medidas institucionales correspondientes: una forma modificada de reconocimiento social se convierte en digna de crédito no sólo cuando es racional desde una perspectiva evaluativa, sino además cuando también cumple la condición de ser justa con la nueva cualidad de valor desde una perspectiva material -algo debe haber cambiado en el mundo físico de los modos de conducta o los hechos institucionales si la persona apelada está efectivamente convencida de que es reconocida de una nueva forma ${ }^{3}$.

Es este segundo componente material el que puede dar ahora la solución para la difícil tarea de diferenciar las formas ideológicas y las justificadas de reconocimiento. Las ideologías del reconocimiento pueden desplegar su poder regulativo, tal como hemos visto, cuando su vocabulario evaluativo es lo suficientemente racional como para descubrir a las personas apeladas, de una manera creíble, una relación consigo mismas nueva y positiva; tales ideologías serán en general tanto más exitosas cuanto de manera más intensa se dirijan a las expectativas evaluativas que apuntan en la dirección de un progreso de la cultura del reconocimiento mutuo. Pero el déficit que posiblemente permite reconocer tales ideologías consiste en la incapacidad estructural para proporcionar las condiciones materiales bajo las cuales son realizables efectivamente las nuevas cualidades de valor de las personas afectadas: entre la promesa evaluativa y el cumplimiento material se abre un abismo, el cual es característico de estas ideologías, porque la provisión de las condiciones institucionales no sería ya compatible con el orden social dominante. Si aplicamos este criterio al ejemplo que expuse anteriormente, se muestra éste, según mi opinión, como consistente: aunque la nueva apelación a los empleados y trabajadores cualificados en tanto que "empresarios de la fuerza de trabajo" contiene la promesa evaluativa de reconocer un grado más elevado de individualidad y motivación intrínseca por el trabajo, sin embargo no procura por sí misma las medidas institucionales que permitirían una realización consistente de los nuevos valores; más bien las personas apeladas son obligadas a simular, en condiciones de trabajo inmodificadas, motivos intrínsecos, flexibilidad y aptitudes aun cuando su proceso de formación no les proporciona los recursos para ello. La nueva forma de reconocimiento no es desde un punto de vista evaluativo deficitaria o irracional, sino que no satisface las

\footnotetext{
${ }^{3}$ En este sentido habla John L. Austin (1972) acerca de que determinadas declaraciones performativas sólo pueden valer como "afortunadas" o cumplidas si en su efecto han hecho "esto o aquello" (p. 72; cf. también la distinción de los "casos desafortunados", p. 24 ss.). En el concepto de "cumplimiento material" traslado aquí su análisis de los enunciados performativos al caso específico del reconocimiento.
} 
exigencias materiales por las que sólo se podría medir íntegramente la credibilidad del reconocimiento justificado; pues las medidas institucionales que serían necesarias para realizar biográficamente el nuevo valor acentuado no son suministradas en el acto de reconocimiento. Pero si ese componente del cumplimiento material es añadido a las condiciones que, al ser consideradas conjuntamente, sustentan la racionalidad del reconocimiento, entonces puede hablarse en relación a las ideologías del reconocimiento de un déficit de racionalidad a un segundo nivel: si bien quisieran ser racionales en el sentido de que toman su vocabulario del ámbito históricamente transformado de las razones evaluativas, sin embargo se muestran como irracionales en tanto que no culminan el acto de reconocimiento más allá del nivel meramente simbólico hasta el cumplimiento material. Un segundo caso de nuevo tipo de reconocimiento que puede mostrarse como ideológico bajo este aspecto es el discurso hoy de moda sobre el "trabajo cívico": también aquí se le concede una distinción simbólica a un grupo social que puede motivar nuevas formas de subyugación voluntaria sin que sean introducidas las correspondientes medidas a nivel institucional.

Claro está que con el criterio formulado no debe inducirse una hermenéutica de la sospecha que siempre está demasiado segura de sí misma. Pues no hay que excluir nunca que entre la promesa evaluativa y el cumplimiento material sólo se abra una distancia temporal que proporciona una demora en la realización de las condiciones institucionales; como en las interacciones simples, también en el nivel del reconocimiento institucional hay que contar frecuentemente con procesos de aprendizaje a largo plazo antes de que el contenido evaluativo de una nueva forma de reconocimiento pueda cristalizar en formas modificadas de conducta o en medidas institucionales. Pero, en resumen, el criterio del cumplimiento material aporta un medio útil para comprobar anticipadamente si una determinada modificación del reconocimiento se trata realmente sólo de un aumento del poder regulador: aquellos modelos institucionales de distinción evaluativa a los que falta toda perspectiva sobre el cumplimiento material podemos denominarlos con buena conciencia como ideologías.

\section{REFERENCIAS BIBLIOGRÁFICAS}

AlthUSSER, L., 1973: "Ideologie und ideologische Staatsapparate (Skizzen für eine Untersuchung)", en Marxismus und Ideologie, VSA, Berlin, p. 111-172 (trad. cast.: "Ideología y aparatos ideológicos del Estado", en Althusser, L., Escritos, Laia, Barcelona, 1974, p. 105-170).

Austin, J. L., 1972: Zur Theorie der Sprechakte (How to do things with Words),

Reclam, Stuttgart (trad. cast.: Cómo hacer cosas con palabras, Paidós, Barcelona, 1982).

ButLeR, J., 2001: "Das Gewissen macht Subjekte aus uns allen. Subjektivation nach Althusser", en Psyche der Macht. Das Subjekt der Unterwerfung, Suhrkamp, 
Frankfurt/Main, p. 101-124 (trad, cast.: Mecanismos psíquicos del poder. Teorías de la sujeción, Cátedra, Madrid, 2001).

Cavell, S., 2002: "Wissen und Anerkenung", en D. Sparti y E. Hammer (eds.), Die Unheimlichkeit des Gewöhnlichen, Fischer, Frankfurt/Main, p. 39-75.

Detel, W., 1998: Macht, Moral, Wissen. Foucault und die klassische Antike, Suhrkamp, Frankfurt/Main.

EMcKe, C., 2000: Kollektive Identitäten. Sozialphilosophische Grunklagen, Campus, New York.

Geuss, R., 1996: Die Idee einer Kritischen Theorie, Syndikat Buchgesellschaft, Bodenheim.

Hausen, K., 1976: “Die Polarisierung der 'Geschlechtscharaktere'. Eine Spiegelung der Dissoziation von Erwerbs- und Familienleben”, en W. Conze (ed.), Sozialgeschichte der Familie in der Neuzeit Europas, Klett, Stuttgart.

Honneth, A., 2000: "Die soziale Dynamik von Mißachtung. Zur Ortsbestimmung einer kritischen Gesellschaftstheorie", en Das Andere der Gerechtigkeit, Suhrkamp, Frankfurt/Main, p. 88-109.

- 2003a: "Umverteilung als Anerkennung. Eine Erwiderung auf Nancy Fraser", en N. Fraser/A. Honneth, Umverteilung oder Anerkennung?, Suhrkamp, Frankfurt/Main, p. 129-224.

- 2003b: "Zwischen Hermeneutik und Hegelianismus" en Unsichtbarkeit. Stationen einer Theorie der Intersubjektivität, Suhrkamp, Frankfurt/Main, p. 106-137.

— 2004a: "Der Grund der Anerkennung. Eine Erwiderung auf kritische Rückfragen", Epílogo a la segunda edición de Kampf um Anerkennung, Suhrkamp, Frankfurt/Main, p. 303-241 (la primera edición de esta obra fue traducida al castellano como La lucha por el reconocimiento, Crítica, Barcelona, 1997).

— 2004b: "Die Unhintergehbarkeit des Fortschritts. Kants Bestimmung des Verhältnisses von Moral und Geschichte", en R. Langthaler (ed.), Recht-Geschichte-Religion. Die Bedeutung Kants für die Philosophie der Gegenwart, Akademie, Berlin.

IKÄHEIMO, H., 2002: “On the Genus and Species of Recognition”, en Inquiry, vol. 45, no 4, p. 447-462.

KanT, I., 1999: Grundlegung zur Metaphysik der Sitten, Felix Meiner, Hamburg (trad. cast.: Fundamentación de la metafísica de las costumbres, Espasa Calpe, Madrid, 1942).

KüHN, B. 1993: "Vom Schalten und Walten der Hausfrau. Hausarbeit in Rat, Tat und Forschung im 19. und 20. Jahrhundert”, en B. Bolognese-Leuchtenmüller y M. Mitterauer (eds.), Frauen-Arbeitswelten. Zur historischen Genese gegenwärtiger Probleme, Verlag für Gesellschaftskritik, Wien, p. 43-66.

LAITINEN, A., 2002: "Interpersonal Recognition: A Response to Value or a Precondition of Personhood?", en Inquiry, vol. 45, no 4, p. 463-478.

LoviBond, E., 2002: Ethical Formation, Harvard University Press, Cambridge.

Margalit, A., 2001: "Recognition II: Recognizing the Brother and the Other", en Aristotelian Society Supplementary, vol. 75, The Aristotelian Society Publications, Bristol, p. 127-139. 
Markell, P., 2003: Bound by Recognition, Princenton University Press, Princenton y Oxford.

MARX, K., 1971 [1867]: Das Kapital. Kritik der politischen Ökonomie, Libro primero (K. Marx/ F. Engels, Werke, Vol. 23), Dietz, Berlin (trad. cast.: El Capital, Siglo XXI, Madrid, 1975).

McDowell, J., 2002: "Zwei Arten von Naturalismus", en Wert und Wirklichkeit. Aufsätze zur Moralphilosophie, Suhrkamp, Frankfurt/Main, p. 30-73.

OlIVIER, K., 2001: Witnessing. Beyond Recognition, University of Minnesota Press, Minneapolis.

OpITZ, S., 2004: Gouvernementabilität im Postfordismus. Macht, Wissen und Techniken des Selbst im Feld unternehmerischer Rationalität, Argument, Hamburg.

RAEITHEL, G., 1995: Geschichte der nordamerikanischen Kultur, Vol 1: Vom Puritanismus bis zum Bürgerkrieg 1600-1860, Zweitausendeins, Frankfurt/Main.

RENAUlt, E., 2004: "Reconnaissance, Institutions, Injustice", en De la reconnaissance, Revue du Mauss, no 23, París, p. 180-195.

RÖSNER, H., 2002: Jenseits normalisierender Anerkennung. Reflexion zum Verhältnis von Macht und Behindertsein, Campus, Frankfurt/Main-New York.

Schilling, R., 2002: Kriegshelden. Deutungsmuster heroischer Männlichkeit in Deutschland 1813-1945, Ferdinand Schöningh, Paderborn.

Verweyst, M., 2000: Das Begehen der Anerkennung. Subjekttheoretische Positionen bei Heidegger, Sartre, Freud und Lacan, Campus, Frankfurt/Main.

Voß, G./PONGRATZ, H.J., 1989: "Der arbeitskraftunternehmer. Eine neue Grundform der Ware Arbeitskraft?”, en Kölner Zeitschrift für Soziologie und Sozialpsychologie, Año 50, Cuaderno 1, p. 131-158. 